value of a single question - "In the past month, how often did you take your medications as the doctor prescribed?" - to predict the risk of adverse cardiovascular events. Nonadherence was defined as taking medication as prescribed $\leq 75 \%$ of the time. Annual follow-up telephone interviews of the patients or their proxy were conducted to identify adverse cardiovascular events (CHD-related death, myocardial infarction or stroke).

A total of $83(8.2 \%)$ of the 1,015 patients included in the final analysis reported medication nonadherence, and 146 (14.4\%) patients experienced adverse cardiovascular events during the mean follow-up time of 3.9 years (range 0.09-5.7 years); 8 patients dropped out of the study. A greater proportion of nonadherent than adherent individuals experienced adverse cardiovascular events $(22.9 \%$ vs $13.7 \% ; P=0.03)$. After multivariate adjustment, the relative rate of cardiovascular events associated with nonadherence (hazard ratio [HR] 2.3, 95\% Cl 1.3-4.3; $P=0.006$ ) was similar to those associated with diabetes mellitus (HR 2.1, 95\% Cl 1.4-3.2; $P<0.001$ ) and current smoking (HR 1.9, 95\% Cl 1.2-3.2; $P=0.009)$.

The authors conclude that a single-item measure of self-reported medication adherence could prove to be a simple method of identifying patients at increased risk of adverse cardiovascular events.

Original article Gehi AK et al. (2007) Self-reported medication adherence and cardiovascular events in patients with stable coronary heart disease: the Heart and Soul Study. Arch Intern Med 167: 1798-1803

\section{Air pollution promotes ischemia and inhibits fibrinolytic function in patients with CHD}

Evidence is accumulating to suggest that exposure to air pollution can increase the chances of developing cardiovascular disease. Little is known, however, about the specific effects air pollution has on individuals with coronary heart disease (CHD) - a population that could be particularly vulnerable to the adverse effects of air pollutants.

In a double-blind crossover study, 20 middleaged men with stable CHD were randomized and exposed to either filtered air or dilute diesel exhaust in two separate hour-long sessions, a minimum of 2 weeks apart. Diesel exhaust was delivered at a concentration of $300 \mu \mathrm{g} / \mathrm{m}^{3}$, which is comparable to pollution levels recorded during heavy traffic in large cities.

On exercise testing during these sessions, heart rate increased similarly in all participants, but a greater maximum ST-segment depression was observed during exposure to diesel exhaust than during exposure to filtered air $(P=0.003)$. Total ischemic burden was also higher during pollution-exposure sessions $(P<0.001)$. Furthermore, the release of endothelial tissue plasminogen activator (induced by intra-arterial bradykinin infusion 6-8h after exposure) was suppressed by $35 \%$ in individuals exposed to diesel exhaust compared with those exposed to filtered air $(P=0.009)$.

This study suggests that even brief exposure to dilute diesel exhaust can exert a dual effect on patients with CHD by worsening myocardial ischemia and inhibiting fibrinolytic capacity. These findings could help to elucidate the underlying mechanisms of the association between air pollution and cardiovascular events.

Original article Mills NL et al. (2007) Ischemic and thrombotic effects of dilute diesel-exhaust inhalation in men with coronary heart disease. $N$ Engl J Med 357: 1075-1082

\section{Fluctuations in triglyceride levels could help to predict CHD risk in young adults}

Evidence suggests that high fasting triglyceride levels could be a risk factor for coronary heart disease (CHD). Triglyceride levels fluctuate with weight and lifestyle changes, however, so triglyceride concentrations at a single time point might not be a reliable indicator of long-term CHD risk.

To investigate whether changes in triglyceride levels over time can improve cardiovascular risk assessment in the young adult, Tirosh et al. performed an observational study of 13,953 apparently healthy adult men with a mean age of 32 years; the participants were not on lipid-lowering medications. Two measurements of fasting serum triglycerides and lifestyle variables were made 5 years apart, and incident cases of angiographically proven CHD were recorded. Low, intermediate and high levels of triglycerides were defined as $\leq 81 \mathrm{mg} / \mathrm{dl} \quad(\leq 0.92 \mathrm{mmol} / \mathrm{l}), 82-130 \mathrm{mg} / \mathrm{dl}$ 